\title{
Structural dissimilarity of large-scale structures in turbulent flows over wavy walls
}

Adrian Zenklusen, Simon Kuhn, and Philipp Rudolf von Rohr

Citation: Phys. Fluids 24, 055112 (2012); doi: 10.1063/1.4719778

View online: http://dx.doi.org/10.1063/1.4719778

View Table of Contents: http://pof.aip.org/resource/1/PHFLE6/v24/i5

Published by the American Institute of Physics.

\section{Related Articles}

Investigations on the local entrainment velocity in a turbulent jet

Phys. Fluids 24, 105110 (2012)

Lagrangian evolution of the invariants of the velocity gradient tensor in a turbulent boundary layer

Phys. Fluids 24, 105104 (2012)

Effects of moderate Reynolds numbers on subsonic round jets with highly disturbed nozzle-exit boundary layers Phys. Fluids 24, 105107 (2012)

Particle transport in a turbulent boundary layer: Non-local closures for particle dispersion tensors accounting for particle-wall interactions

Phys. Fluids 24, 103304 (2012)

Convection and reaction in a diffusive boundary layer in a porous medium: Nonlinear dynamics

Chaos 22, $037113(2012)$

\section{Additional information on Phys. Fluids}

Journal Homepage: http://pof.aip.org/

Journal Information: http://pof.aip.org/about/about_the_journal

Top downloads: http://pof.aip.org/features/most_downloaded

Information for Authors: http://pof.aip.org/authors

\section{ADVERTISEMENT}

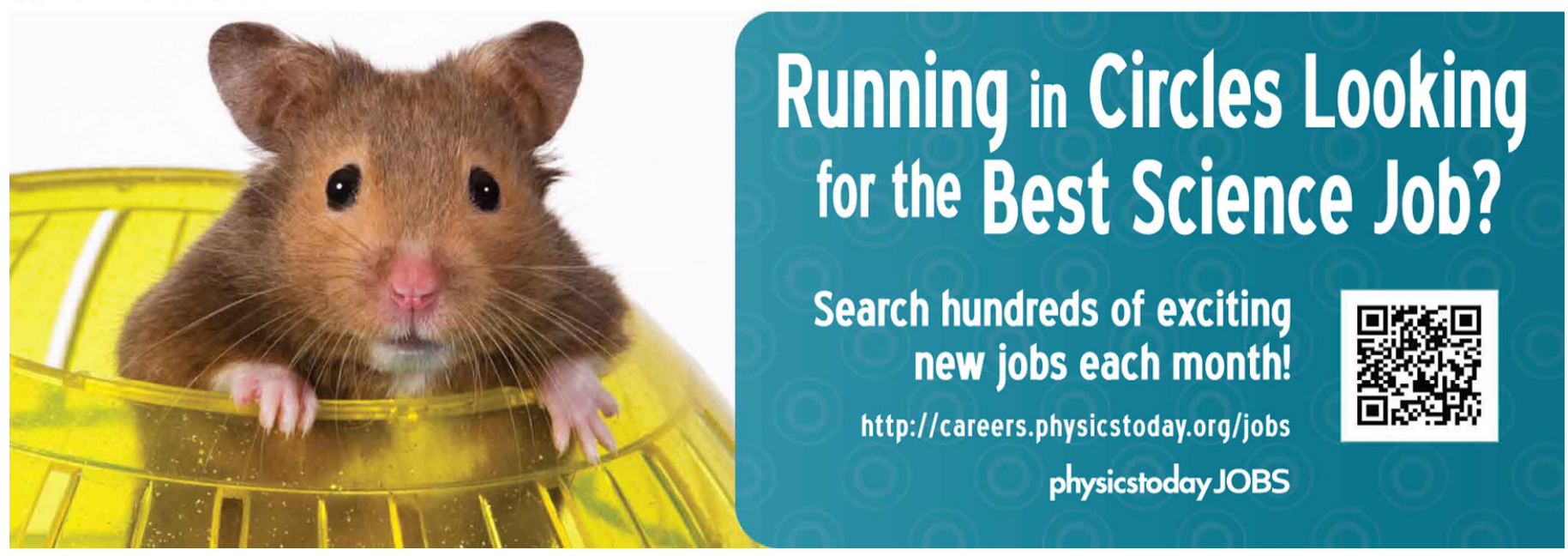




\title{
Structural dissimilarity of large-scale structures in turbulent flows over wavy walls
}

\author{
Adrian Zenklusen, ${ }^{1}$ Simon Kuhn, ${ }^{2}$ and Philipp Rudolf von Rohr ${ }^{1, \text { a) }}$ \\ ${ }^{1}$ Institute of Process Engineering, ETH Zurich, CH-8092 Zurich, Switzerland \\ ${ }^{2}$ Department of Chemical Engineering, Massachusetts Institute of Technology, Cambridge, \\ Massachusetts 02139, USA
}

(Received 8 December 2011; accepted 16 April 2012; published online 30 May 2012)

\begin{abstract}
Turbulent flows over a wavy wall are investigated in channels with a wavy bottom and a flat top with different channel heights. Flow structures are determined from proper orthogonal decompositions of velocity fields measured with particle image velocimetry. Three different channel heights are considered, which are characterized by blockage ratios $\beta$ (half channel height to wave amplitude ratio) 3.3, 6.7, and 10 . Measurements are evaluated at comparable Reynolds numbers $(R e)$ around 10000. Structural similarity of large-scale structures, which is valid at $\beta=6.7$ and 10 , no longer holds at $\beta=3.3$. Furthermore, characteristic regions of flows over wavy walls exhibit different locations in the case of the smallest channel height. @ 2012 American Institute of Physics. [http://dx.doi.org/10.1063/1.4719778]
\end{abstract}

\section{INTRODUCTION}

Turbulent flows over rough surfaces or obstacles are of interest in many technical and geophysical situations. Roughness or obstacles add a degree of complexity to the flow over a flat surface, which induces increased heat, mass, and momentum transport. In many studies a wavy wall is chosen as a reference case. The turbulent flow over a wavy wall exhibits many characteristics of the above described flow, such as flow separation, flow reattachment, and streamline curvature. Furthermore, the geometry of the wavy wall is well defined by a sinusoidal curve, which is characterized by the amplitude ( $a$ ) to wavelength $(\lambda)$ ratio $\alpha$

$$
\alpha=\frac{2 a}{\lambda} .
$$

Flow studies in water channels or wind tunnels with a wavy bottom wall and a flat top wall have been conducted since the thirties of the last century. ${ }^{1,2}$ The focus of early works was on the description of non-separated flows over small amplitude waves $(\alpha<0.03)$ by linear stability analysis. ${ }^{3,4}$ With increased $\alpha$ the flow starts to separate and the description with linear stability analysis becomes insufficient. For the separated flow over waves three characteristic regions are identified, ${ }^{5,6}$ which are the separated region itself, and the regions of maximum positive and maximum negative Reynolds shear stress. The influence of changes in the wave amplitude on the turbulent flow over solid waves has been partly addressed in earlier studies. ${ }^{3,7}$ Raupach et al. ${ }^{8}$ hypothesized that the outer flow over rough surfaces has a universal character and is independent of the interaction with the wall. ${ }^{8}$ Scaling the turbulence properties in the outer flow with the friction velocity, similarities between flat and wavy surfaces have been observed. ${ }^{9,10}$

Based on the observation of streamwise-oriented large-scale structures by Miller ${ }^{11}$ and Gong et al. ${ }^{12}$ Günther and Rudolf von Rohr ${ }^{13}$ applied proper orthogonal decomposition (POD) to quantitatively investigate large-scale structures in a developed flow over a wavy wall. This study revealed dominant eigenfunctions with a characteristic spanwise scale of $1.5 \lambda$ at laminar and turbulent flow

\footnotetext{
a)vonrohr@ipe.mavt.ethz.ch.
} 
conditions. The energy content of the two most dominant modes is found to be almost 50\%, and these large-scale structures were found to meander laterally. ${ }^{14}$

The research on large-scale structures in flows over wavy walls and their impact on momentum and scalar transport has been conducted further at our laboratory: Large-scale structures were also observed in temperature fields of flows over heated wavy walls, ${ }^{15}$ and quantitative agreement between these large-scale thermal and momentum structures was found. ${ }^{16}$ Considering the outer flow over different wavy walls, structural similarity of the large-scale structures was found. ${ }^{17,18}$ The two most dominant large-scale structures of the different wave profiles showed a characteristic spanwise scale of $1.5 H$ (where $H$ denotes the channel height). These studies showed that the outer variable to scale the most dominant eigenfunctions is the channel height $H$, not the wavelength $\lambda$, as proposed earlier. ${ }^{13}$ Furthermore, the effect of large-scale structures on mass $\operatorname{transfer}^{18,19}$ and the role of large-scale structures in mixed convective flows over solid waves were investigated. ${ }^{20}$ These experimental works from our laboratory were further extended by numerical studies extracting structural information of wavy wall flows. ${ }^{21,22}$

The major finding of our research is the presence of large-scale structures, which are found for each flow case and which are directly linked to the transport mechanisms of momentum and scalars. POD on large-scale structures have revealed that the two most dominant flow structures are structurally similar for different wavy flow situations, in a sense that POD eigenfunctions show a characteristic spanwise scale of $1.5 \mathrm{H}$. All flow studies for wavy walls of our laboratory were conducted in the regime of flows over obstacles, in which similarities are less expected than in flows over rough surfaces.

The question arises if structural similarity holds in channels, where the obstacle size is more substantial, such as in narrow channels with wavy walls. Therefore, the channel height was varied for flows over a wavy wall $(\alpha=0.1)$ at comparable $R e$. The blockage ratio $\beta$, which characterizes the ratio of the channel height to the roughness, is defined as

$$
\beta=\frac{H}{2 a},
$$

where $H$ is the channel height. Structural similarity in dependence of the characteristic length scale was investigated by Natrajan et al. ${ }^{23}$ in the case of turbulent capillary/pipe flows. In this study hairpin-like structures and hairpin vortex packets are structurally similar for micro- and macroscale wall turbulence.

In this paper, we present results of flows over a wavy wall $(\alpha=0.1)$ with different $\beta$ (3.3, $6.7,10)$ at comparable $R e$, which enables us to systematically address the effects of flow blockage on large-scale structures. In addition to the characterization of the large-scale structures, we also focused on the characteristic regions of flows over wavy walls to achieve a more complete picture of the present flow situation. The results contribute to the understanding of large-scale structures and characteristic zones in narrow wavy channels.

\section{FLOW SITUATION}

In Fig. 1 the flow over a wavy bottom wall is drawn schematically with its characteristic regions. The coordinate in streamwise direction is $x_{1}, x_{2}$ is normal to the wall and $x_{3}$ points in the spanwise direction. The corresponding velocity components are $u_{1}, u_{2}$, and $u_{3}$.

The flow is characterized by the Reynolds number

$$
R e=\frac{u_{b} \cdot H}{2 v},
$$

where $H$ is the height of the channel and $v$ denotes the kinematic viscosity. $u_{b}$ is the bulk velocity and is defined as

$$
u_{b}=\frac{\int_{0}^{\lambda} \int_{x_{2, w}}^{H} u_{1}\left(x_{1}, x_{2}\right) \mathrm{d} x_{2} \mathrm{~d} x_{1}}{H \lambda} .
$$




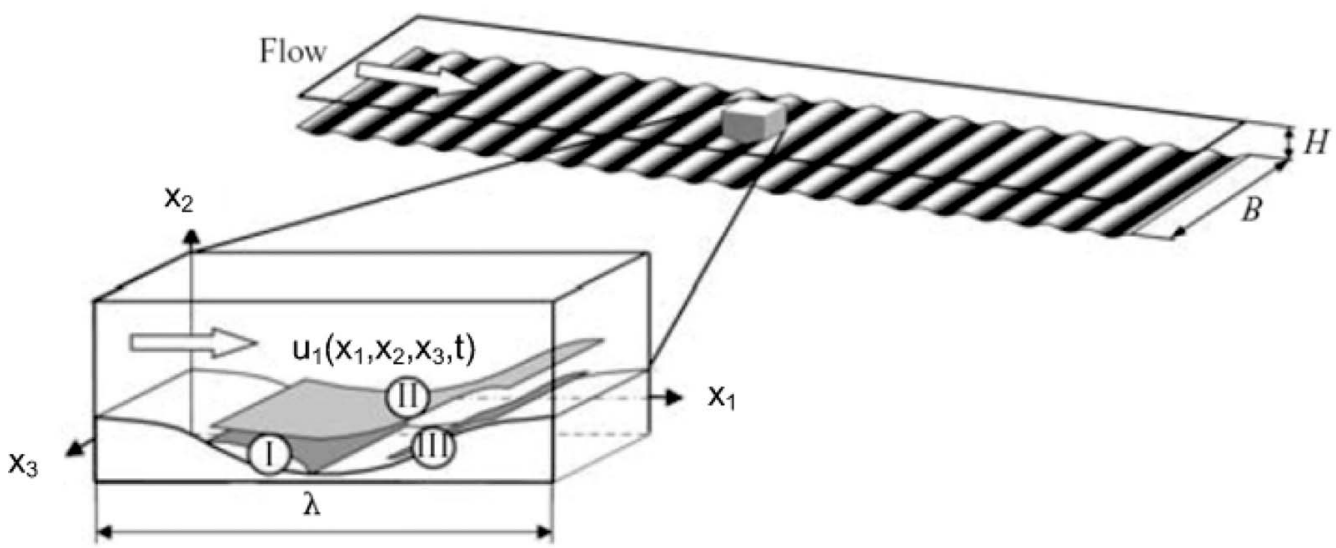

FIG. 1. Flow over a wavy wall. Characteristic regions: $I$ separation region, $I I$ maximum positive Reynolds shear stress, and III maximum negative Reynolds shear stress.

The wavy wall profile is described by

$$
x_{2, w}\left(x_{1}\right)=a \cdot \cos \left(\frac{2 \pi x_{1}}{\lambda}\right),
$$

where $a$ is the wave amplitude and $\lambda$ is the wavelength.

Three characteristic regions of flows over wavy walls were defined by Cherukat et al. ${ }^{5}$ and Henn and Sykes. ${ }^{6}$ In Fig. 1, region I is the separation region, and II and III, respectively, are regions of maximum positive and maximum negative Reynolds shear stresses $\left(-\rho \overline{u_{1}^{\prime} u_{2}^{\prime}}\right)$. The flow separates due to too large positive pressure gradients, which are introduced by streamline expansions over large wave amplitudes $(\alpha>0.03)$. The shear layer, introduced by the separation, is bounded by the characteristic regions II and III and is the location of turbulence production.

Large-scale structures of flows over wavy walls are first identified by Miller ${ }^{11}$ and Gong et al. $^{12}$ as streamwise-oriented, counter-rotating vortices. A quantitative description based on POD has revealed that the two most dominant structures have spanwise characteristic length scales of $1.5 \mathrm{H}$. The characteristic spanwise length scale has been confirmed for different amplitude-to-wavelength ratios $\alpha$ (Refs. 17 and 18) and for a large range of $R e$ between laminar and turbulent flow conditions. ${ }^{13}$

\section{EXPERIMENTS}

Experiments were performed in the water channel facility pictured in Fig. 2. The wavy surface is represented by a wavy bottom wall in the test section with a wavelength of $30 \mathrm{~mm}$ and a wave amplitude of $1.5 \mathrm{~mm}(\alpha=0.1)$. The top wall of the test section is flat and experiments were conducted for channel heights $H$ of 10,20 , and $30 \mathrm{~mm}(\beta=3.3,6.7,10)$. The test section is $2150 \mathrm{~mm}$ long and

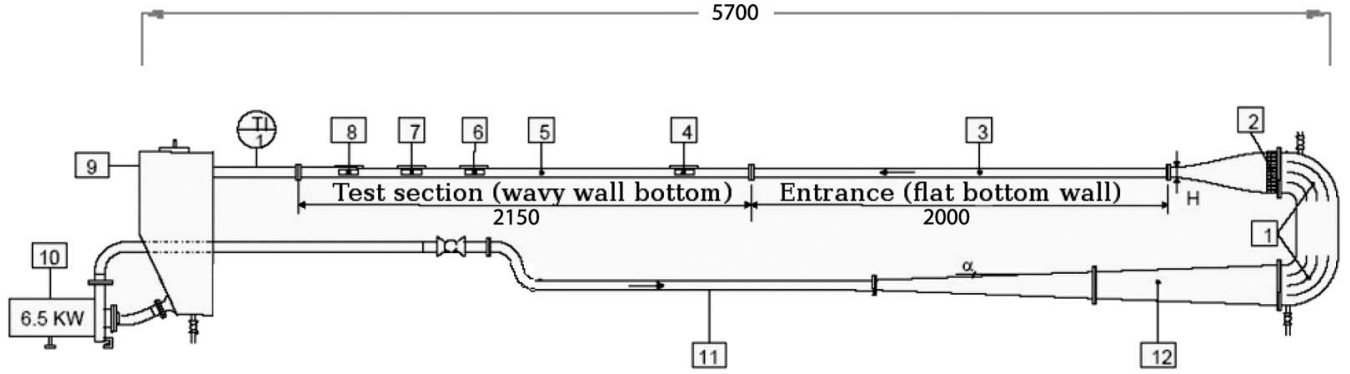

FIG. 2. Water channel facility. (1) Turning elbows, (2) honeycomb, (3) flat-walled entrance channel, (5) section with wavy bottom, (4, 6-8) optical view ports, (9) reservoir, (10) frequency-controlled pump, (11) pipe, and (12) diffusor. Adapted from Günther and Rudolf von Rohr. ${ }^{13}$ 
TABLE I. Field of view (FOV) and spatial resolutions of the different measurements used for structural information. The vector grid spacing is the resolution without $50 \%$ overlap of the interrogation areas.

\begin{tabular}{lrrc}
\hline \hline & Plane & \multicolumn{1}{c}{ FOV } & Resolution (vector grid spacing) \\
\hline$\beta=3.3$ & $x_{1}, x_{2}$ & $3 H \times 1.4 H$ & $0.78 \mathrm{~mm} \times 0.78 \mathrm{~mm}$ \\
$\beta=3.3$ & $x_{1}, x_{3}$ & $4.1 H \times 6.2 H$ & $1.48 \mathrm{~mm} \times 1.48 \mathrm{~mm}$ \\
$\beta=6.7$ & $x_{1}, x_{2}$ & $1.5 H \times 1.2 H$ & $0.84 \mathrm{~mm} \times 0.84 \mathrm{~mm}$ \\
$\beta=6.7$ & $x_{1}, x_{3}$ & $2.65 H \times 3.5 H$ & $1.68 \mathrm{~mm} \times 1.7 \mathrm{~mm}$ \\
$\beta=10$ & $x_{1}, x_{2}$ & $1 H \times 1.1 H$ & $1.08 \mathrm{~mm} \times 1.1 \mathrm{~mm}$ \\
$\beta=10$ & $x_{1}, x_{3}$ & $2.27 \mathrm{H} \times 2.9 \mathrm{H}$ & $2.16 \mathrm{~mm} \times 2.2 \mathrm{~mm}$ \\
\hline \hline
\end{tabular}

the width of the channel $B$ is $360 \mathrm{~mm}$. The channel heights were adjusted by shifting the position of the test section's bottom. The intersection between the entrance channel (height $30 \mathrm{~mm}$, length $2000 \mathrm{~mm}$, width $360 \mathrm{~mm}$ ) and the test section was therefore modified by inserting chokes of angles of $2^{\circ}$ in order to prevent flow separation. Experiments were conducted at $R e$ up to 10900 ( $R e=8600$ for $\beta=3.3, R e=9200$ for $\beta=6.7, R e=10900$ for $\beta=10$ ) with deionized water as working fluid, which results in the following bulk velocities $u_{b}(1.62 \mathrm{~m} / \mathrm{s}$ for $\beta=3.3,0.96 \mathrm{~m} / \mathrm{s}$ for $\beta=6.7,0.69 \mathrm{~m} / \mathrm{s}$ for $\beta=10$ ).

Particle image velocimetry (PIV) was applied to measure velocity fields in planes parallel to the sidewalls and parallel to the top wall. Optical access through the sidewalls and the top wall is provided at three different positions in the test section. Measurements are conducted for channel heights 10 and $20 \mathrm{~mm}$ after the 40th wave crest and after the 50th wave crest, respectively, for the channel height $30 \mathrm{~mm}$ in $\left(x_{1}, x_{2}\right)$ - and $\left(x_{1}, x_{3}\right)$-planes. One thousand image pairs were recorded in all measurements by a CCD camera with a pixel resolution of $1344 \times 1024$ pixels $^{2}$ at a frame rate of $4 \mathrm{~Hz}$. As light source a Nd:YAG dual pulsed laser was used. The flow was seeded by hollow glass spheres of $10 \mu \mathrm{m}$ in diameter (density: $1100 \mathrm{~kg} \mathrm{~m}^{-3}$ ). The sizes of the field of view (FOV) of different measurements are given in Table I. For the subsequent adaptive cross correlations these FOVs are subdivided in interrogation areas of $32 \times 32$ pixels $^{2}$. The adaptive correlation of Dantec's DynamicStudio ${ }^{\circledR}$ was used to calculate velocity vectors. Initial interrogation area sizes of $64 \times 64$ pixels $^{2}$ are used, which are bisected for subsequent iterations. An overlap of $50 \%$ is applied to all interrogation areas and spurious vectors are detected by local neighborhood validation based on velocity gradients and are replaced by a local interpolation scheme. For the investigation of the characteristic zones, an additional adaptive cross correlation step was performed to increase the resolution of the near-wall regions, which results in a final interrogation area size of $16 \times 16$ pixel. Detailed information about FOVs and spatial resolutions of the different measurements are summarized in Table I.

Sampling errors are estimated by a bootstrap analysis. For every measurement, the spatiotemporal statistics are calculated from 800 randomly chosen velocity fields (out of 1000), and this procedure is repeated 350 times. A confidence level, which is defined by twice the standard deviation of the so obtained spatio-temporal turbulent statistics, was determined to estimate the uncertainty of the rms velocity component. This method yields the following uncertainties for the data used to examine large-scale structures: for $u_{1, r m s}$ in $\left(x_{1}, x_{2}\right)$-plane $6 \%(\beta=3.3), 5.14 \%(\beta=6.7), 4.86 \%$ $(\beta=10)$, and for $u_{1, r m s}$ in the $\left(x_{1}, x_{3}\right)$-plane $5.14 \%(\beta=3.3), 5.43 \%(\beta=6.7), 4 \%(\beta=10)$. Uncertainties of the data to investigate the characteristic zones are: for $\overline{u_{1}} 5.71 \%(\beta=3.3), 5.14 \%$ $(\beta=6.7), 4.86 \%(\beta=10)$ and for $\overline{u_{1}^{\prime} u_{2}^{\prime}} 3.71 \%(\beta=3.3), 4.29 \%(\beta=6.7), 4.29 \%(\beta=10)$.

For flows over wavy walls with comparable Reynolds number and similar experimental equipment as used in this study, the uncertainty of the PIV method was estimated to be in the order of $1 \%{ }^{24}$

\section{RESULTS}

Flow structures are extracted by performing a POD or a Karhunen-Loeve decomposition of the flow field. The idea of POD is to describe a given statistical ensemble through a minimal number of modes. The method of snapshots ${ }^{25}$ is applied on the streamwise velocity component $u_{1}$. 
Reynolds averaging is applied and the streamwise velocity component $u_{1}$ measured in the $\left(x_{i}, x_{j}\right)$-plane is decomposed into a mean and fluctuating part

$$
u_{1}\left(x_{i}, x_{j}, t\right)=\overline{u_{1}}\left(x_{i}, x_{j}\right)+u_{1}^{\prime}\left(x_{i}, x_{j}, t\right) .
$$

We consider discrete times $t_{i}$ with $i=1, \ldots, M$, and $1, \ldots, N$ discrete locations in the $\left(x_{i}, x_{j}\right)$-plane, where $N=m n$ with $x_{i}:(1, m)$ and $x_{j}:(1, n)$. The resulting set of spatio-temporal data can be written as the $(N \times M)$ matrix for $u_{1}^{\prime}$ :

$$
\mathbf{M}=\left[\begin{array}{cccc}
u_{1,11}^{\prime} & u_{1,12}^{\prime} & \ldots & u_{1,1 M}^{\prime} \\
u_{1,21}^{\prime} & u_{1,22}^{\prime} & \ldots & u_{1,2 M}^{\prime} \\
\ldots & \ldots & \ldots & \ldots \\
u_{1, N 1}^{\prime} & u_{1, N 2}^{\prime} & \ldots & u_{1, N M}^{\prime}
\end{array}\right]
$$

Using the method of snapshots, the $(M \times M)$ covariance matrix reads

$$
\mathbf{C}_{i j}=\frac{1}{M}\left\langle\mathbf{M}_{i} \mathbf{M}_{j}\right\rangle, \quad i, j=1, \ldots, M,
$$

where $\langle\cdot, \cdot\rangle$ denotes the Euclidean inner product. Since this covariance matrix is symmetric its eigenvalues, $\lambda_{i}(i=1, \ldots, M)$, are non-negative, and its eigenvectors, $\phi_{i}(i=1, \ldots, M)$, form a complete orthogonal set. The orthogonal eigenfunctions are

$$
\Pi^{[k]}=\sum_{i=1}^{M} \phi_{i}^{[k]} \mathbf{M}_{i}, \quad k=1, \ldots, M,
$$

where $\phi_{i}^{[k]}$ is the $i$ th component of the $k$ th eigenvector. The total energy $E$ of the flow is obtained by the summation over the eigenvalues $\lambda_{i}(i=1, \ldots, M)$

$$
E=\sum_{i=1}^{M} \lambda_{i}
$$

The fractional contribution of each eigenfunction to the total energy of $u_{1}^{\prime}$ is given by the fractional contribution of its associated eigenvalue

$$
\frac{E_{k}}{E}=\frac{\lambda_{k}}{\sum_{i=1}^{M} \lambda_{i}}
$$

Using only the first $K$ most energetic eigenfunctions an approximation of the original data is given by

$$
u_{1}=\overline{u_{1}}+\sum_{i=1}^{K} a_{i} \Pi^{[i]}
$$

where the coefficients $a_{i}$ are calculated from the projection of the sample vector $u_{1}^{\prime}$ onto the eigenfunction $\Pi^{[i]}$ :

$$
a_{i}=\frac{u_{1}^{\prime} \cdot \Pi^{[i]}}{\Pi^{[i]} \cdot \Pi^{[i]}} .
$$

This operation corresponds to low-pass filtering and can be used to study the structure of the largescale structures. Structural similarity is usually identified using two-point correlation functions, but it was shown that the covariance matrix yields the same dominant spatial modes as the two-point correlation matrix. ${ }^{25}$ The eigenproblem of the covariance matrix is a much smaller and computationally more tractable problem.

\section{A. Large-scale structures in the $\left(x_{1}, x_{2}\right)$-plane}

$u_{1}$ and $u_{2}$ are the most dominant velocity components for flows over wavy walls. Structural information from the $\left(x_{1}, x_{2}\right)$-plane is therefore essential to describe the most dominant, most energetic 


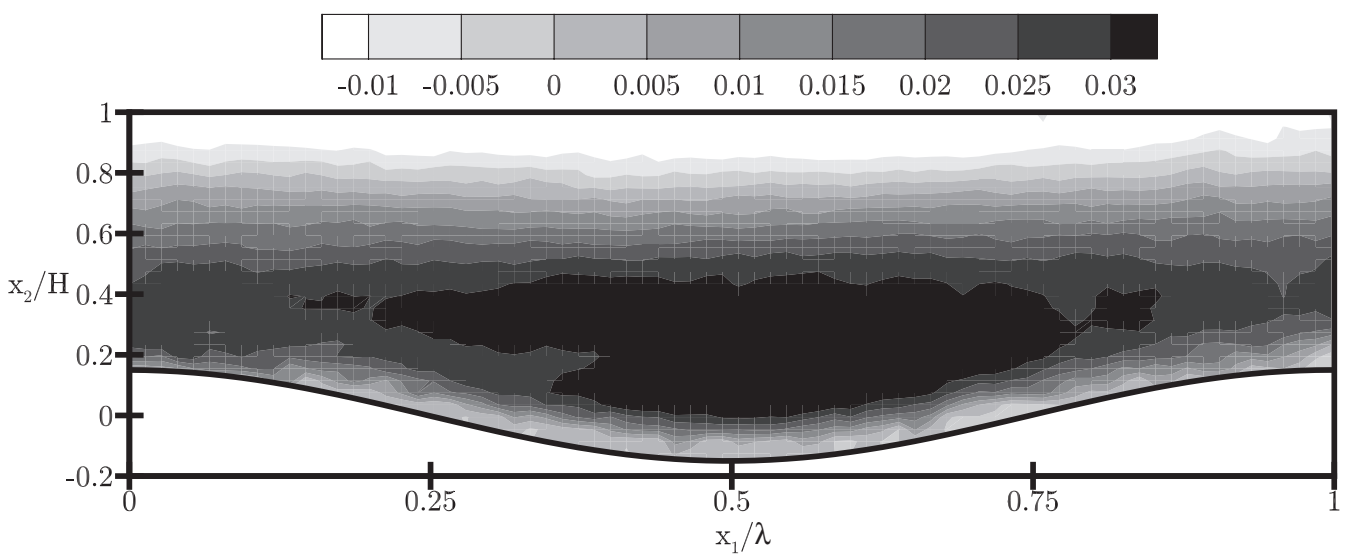

(a)

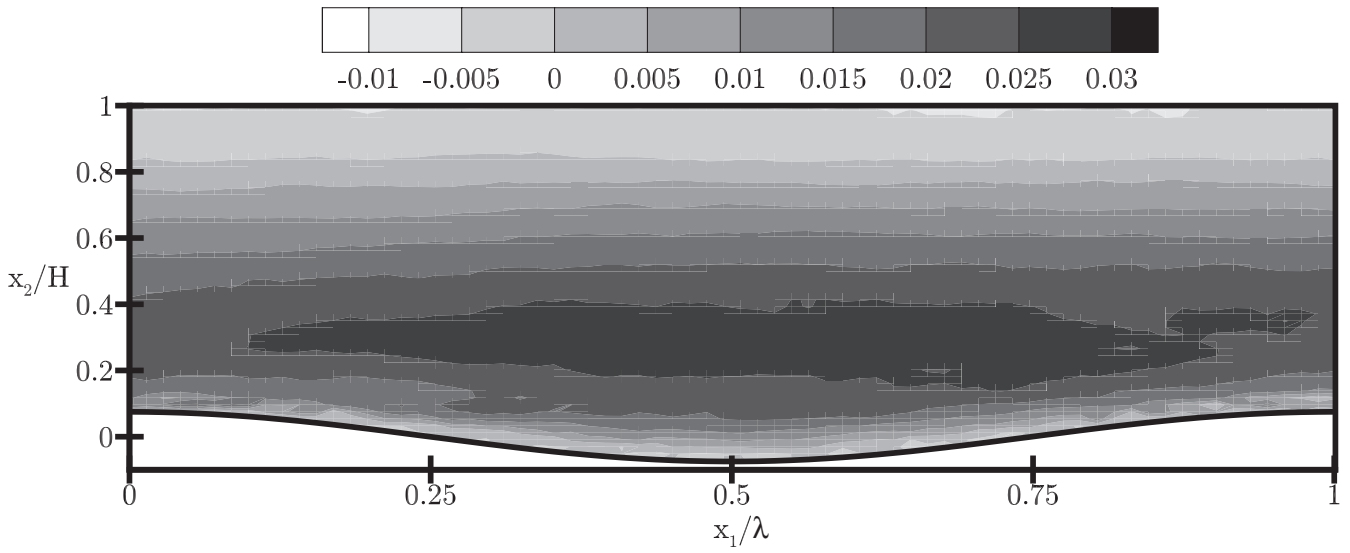

(b)

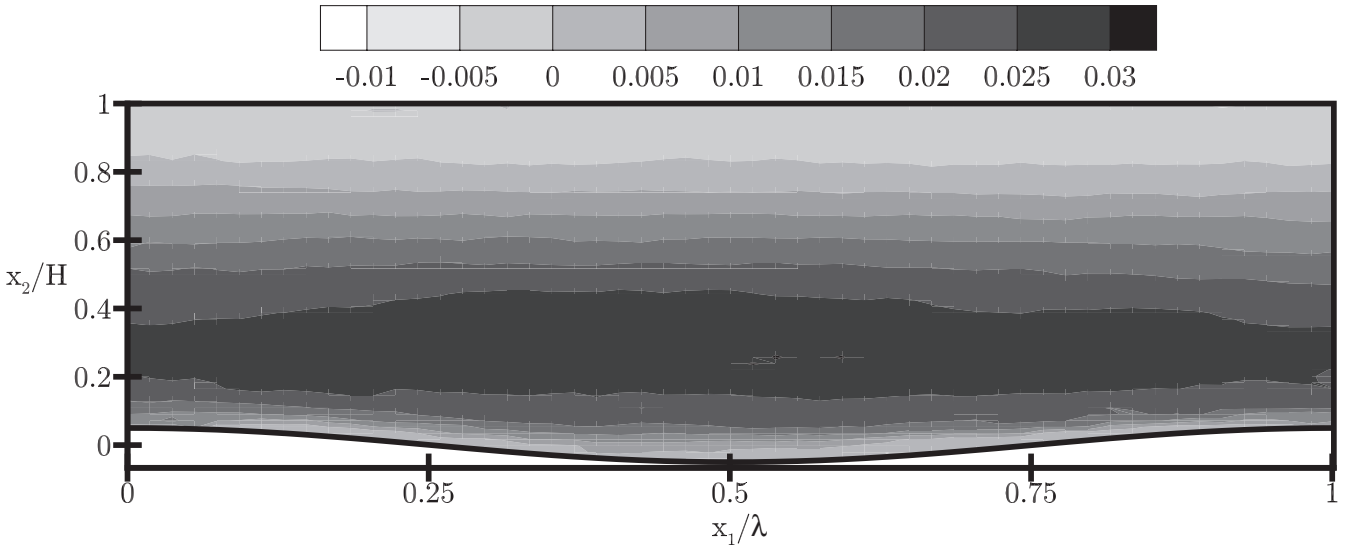

(c)

FIG. 3. First eigenfunctions of $u_{1}^{\prime} / u_{b}$. (a) $\Pi_{1, u_{1}^{\prime} / u_{b}}$ at $\operatorname{Re} 8600$ at $\beta=3.3$. (b) $\Pi_{1, u_{1}^{\prime} / u_{b}}$ at $\operatorname{Re} 9200$ at $\beta=6.7$. (c) $\Pi_{1, u_{1}^{\prime} / u_{b}}$ at $\operatorname{Re} 10900$ at $\beta=10$. All slices are located in the center of the channel.

flow structures, which are obtained by performing PODs on $u_{1}^{\prime} / u_{b}$ in $\left(x_{1}, x_{2}\right)$-planes located in the center of the channel. In Figs. 3(a)-3(c) the eigenfunction of the most dominant (or first) eigenmode of the streamwise velocity normalized by the bulk velocity, $\Pi_{1, u_{1}^{\prime} / u_{b}}$, is presented for the three different channel heights at comparable $R e$. The position of the maximum is shifted towards the wavy wall for decreasing $\beta$. 

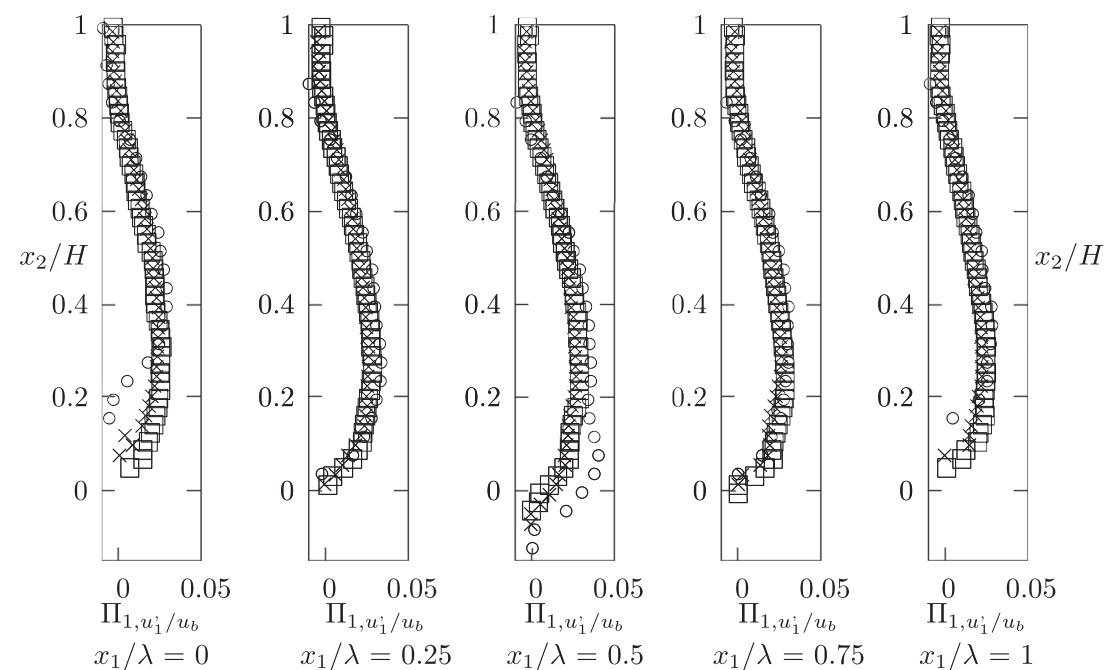

FIG. 4. First eigenfunctions of $u_{1}^{\prime} / u_{b}, \Pi_{1, u_{1}^{\prime} / u_{b}}$ for comparable Re. " $\bigcirc$ " $\beta=3.3$, “×” $\beta=6.7$, “ $\square$ " $\beta=10$.

Profiles of $\Pi_{1, u_{1}^{\prime} / u_{b}}$ at different positions along $x_{1}$ are given in Fig. 4. The profiles of $\beta=6.7$ and 10 are similar when they are scaled with the channel height. However, the profile of $\beta=3.3$ is different to the other blockage factors at the wave crests and the wave trough. Especially in the wave trough the maximum of the profile is shifted towards the wavy wall. The observed dissimilarity between the first eigenfunctions at $\beta=6.7$ and 10 and the first eigenfunction of $\beta=3.3$ shows structural dissimilarity of the most energetic large-scale structure.

In Fig. 5, profiles of the second most energetic eigenfunctions are presented. Similarity is observed for the cases $\beta=10$ and $\beta=6.7$ for the eigenfunctions of the second most energetic eigenmodes. In the case with the smallest channel height the profile is completely different compared to the other cases.

The fractional energy contributions of the eigenmodes $\lambda_{i}$ of the POD modes of $u_{1}^{\prime} / u_{b}$ are plotted in Fig. 6. The energy contributions among the different modes are similar for $\beta=10$ and $\beta=6.7$. The most dominant eigenmode of $u_{1}^{\prime} / u_{b}$ accounts for around $30 \%$ of the energy in these two
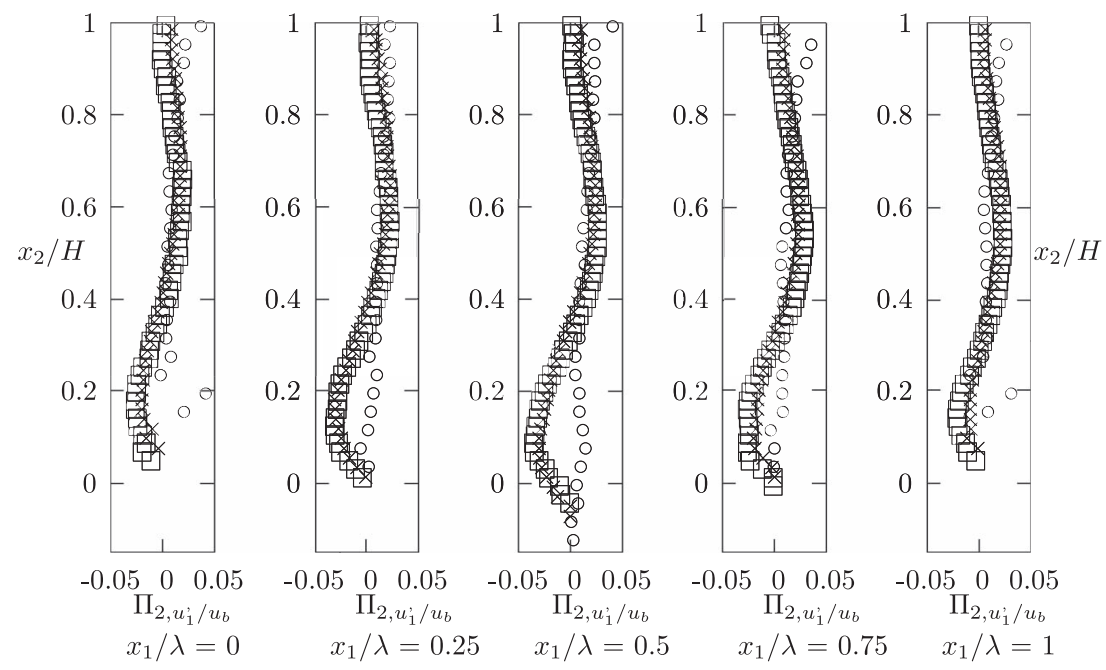

FIG. 5. Second eigenfunctions of $u_{1}^{\prime} / u_{b}, \Pi_{2, u_{1}^{\prime} / u_{b}}$ for comparable $R e$. " $\bigcirc$ " $\beta=3.3$, “×” $\beta=6.7$, “ $\square$ " $\beta=10$. 


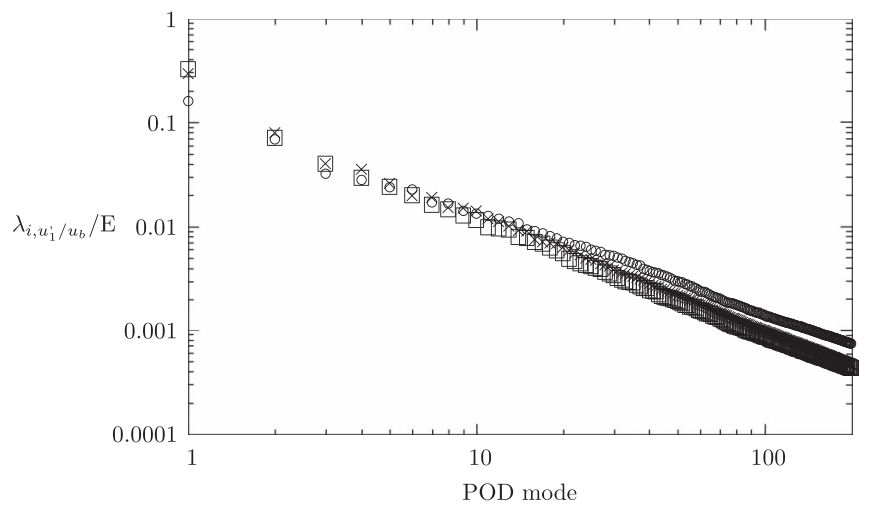

FIG. 6. Eigenvalues of $u_{1}^{\prime} / u_{b}, \lambda_{i, u_{1}^{\prime} / u_{b}}$ for comparable Re. " $\bigcirc " \beta=3.3$, " $\times ” \beta=6.7$, “ $\square$ " $\beta=10$.

cases. Around $45 \%$ of the energy is given by the four most dominant eigenmode. For $\beta=3.3$ the eigenvalue spectrum is broader, so the most energetic mode of $u_{1}^{\prime} / u_{b}$ accounts for $16 \%$ of the total energy and the four most dominant modes around $30 \%$. The energy contribution of the higher modes is increased compared to the cases $\beta=6.7$ and $\beta=10$, meaning that the small scale structures contain more energy and are more relevant for the flow. Also this dissimilarity between the spectra of eigenvalues of $\beta=6.7$ and $\beta=10$, and the spectrum of eigenvalues of $\beta=3.3$ is evidence of structural dissimilarity of large-scale structures.

\section{B. Large-scale structures in the $\left(x_{1}, x_{3}\right)$-plane}

The measurement locations in the $\left(x_{1}, x_{3}\right)$-planes are chosen to match the maximum of the $\Pi_{1, u_{1}^{\prime} / u_{b}}$ profiles in the $\left(x_{1}, x_{2}\right)$-planes. For $\beta=6.7$ and 10 these maximum positions are found at $0.26 H$ from the wavy wall. In the case of $\beta=3.3$, the maximum value of $\Pi_{1, u_{1}^{\prime} / u_{b}}$ is found in the wave trough; therefore, we did chose a location of $0.32 \mathrm{H}$, which represents the maximum above the entire wavy surface. The $\left(x_{1}, x_{3}\right)$-planes are obtained at slices, located in the center of the channel.

Figures 7(a)-7(c) show the first eigenfunction in streamwise direction normalized by the corresponding bulk velocity, $\Pi_{1, u_{1}^{\prime} / u_{b}}$, for $\beta=3.3,6.7$, and 10 .

To quantitatively describe the characteristic spanwise length scales $\Lambda_{1, x_{3}}$ of the first eigenmode its profiles are presented in Fig. 8 for different $\beta$ by streamwise averaging $[\cdot]$ and shifting $\Pi_{1, u_{1}^{\prime} / u_{b}}$ in spanwise direction. $\Lambda_{1, x_{3}}$ corresponds to $1.5 H$ for $\beta=10$, in the intermediate case of $\beta=6.7 \Lambda_{1, x_{3}}$ increases to $1.7 \mathrm{H}$, and for the smallest channel height this spanwise scale is $2.9 \mathrm{H}$. For $\beta=6.7$ and $\beta$ $=10, \Lambda_{1, x_{3}}$ is around $1.5 H$, whereas at $\beta=3.3$ this spanwise scale deviates almost by a factor of 2 . The scaling with $H$ does not hold anymore at $\beta=3.3$, which in turn implies structural dissimilarity of the most energetic large-scale structure. The eigenfunctions of the second eigenmodes of the $\left(x_{1}, x_{3}\right)$-plane are considered in Fig. 9. For the second eigenmode, characteristic spanwise length scales $\Lambda_{2, x_{3}}$ for $\beta=10$ and 6.7 are $1.4 H$ and $1.8 H$, respectively. However, this characteristic length scale for $\beta=3.3$ is $2.6 \mathrm{H}$. Here, the trend of the first eigenmode with similar characteristic length scales for $\beta=6.7$ and 10 around $1.5 \mathrm{H}$ is confirmed, which again deviates in the case of $\beta=3.3$. Also for the second eigenmode, it is concluded that it exhibits structural changes.

The eigenvalues $\lambda_{i, u_{1}^{\prime} / u_{b}}$ of the $\left(x_{1}, x_{3}\right)$-plane are presented in Fig. 10. Again, for $\beta=6.7$ and 10 the eigenvalue spectra are similar. The most energetic eigenmode accounts for roughly $15 \%$ of the energy and the four most energetic eigenmodes contribute to around $40 \%$. For the smallest channel height ( $\beta=3.3$ ), the most energetic eigenmode accounts for $7 \%$ and the four most energetic for around $25 \%$ of the energy. And also in the $\left(x_{1}, x_{3}\right)$-plane the eigenvalue spectra for $\beta=6.7$ and $\beta=10$ are similar and deviations are observed for $\beta=3.3$. Thus, the structural similarity of large-scale structures in the outer flow is not anymore given. 
(a)

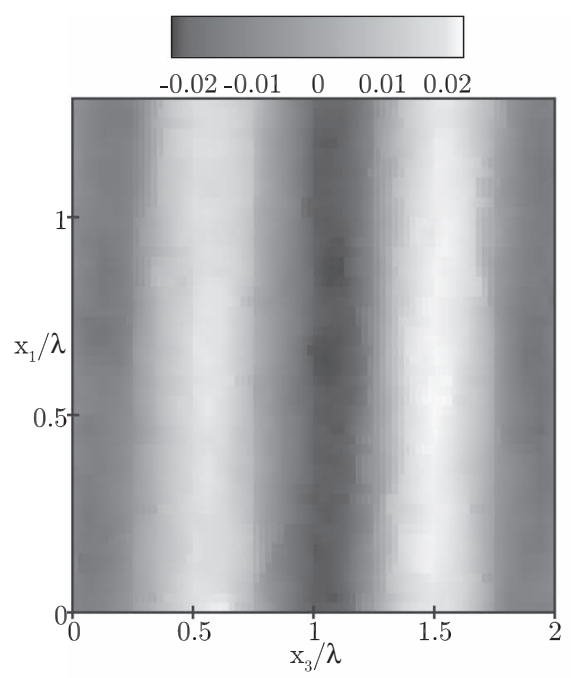

(b)

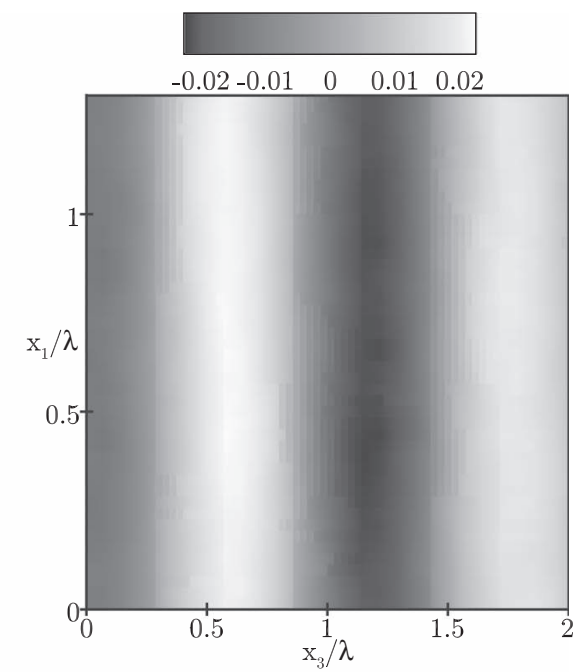

(c)

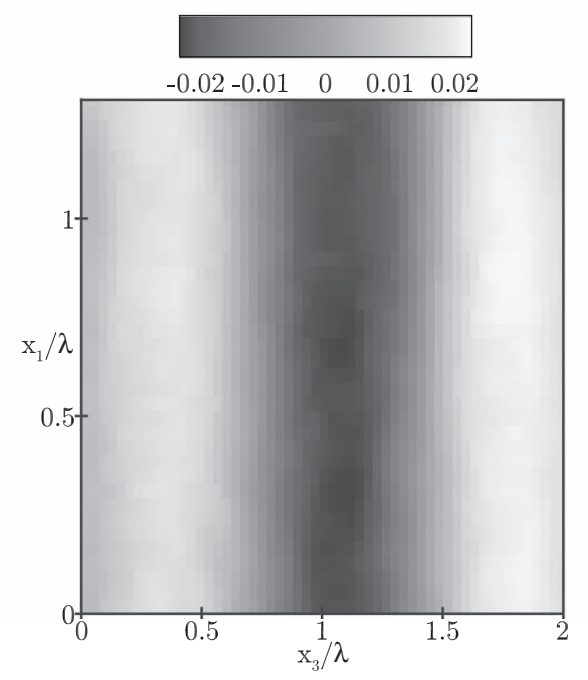

FIG. 7. (a) $\Pi_{1, u_{1}^{\prime} / u_{b}}$ at $R e=8600$ at $\beta=3.3$. (b) $\Pi_{1, u_{1}^{\prime} / u_{b}}$ at $R e=9200$ at $\beta=6.7$. (c) $\Pi_{1, u_{1}^{\prime} / u_{b}}$ at $R e=10900$ at $\beta=10$.

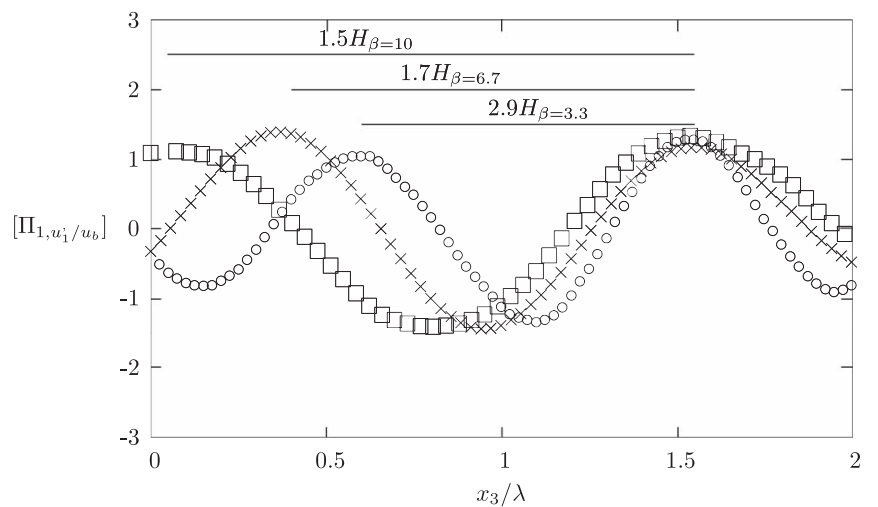

FIG. 8. First eigenfunctions of $u_{1}^{\prime} / u_{b}, \Pi_{1, u_{1}^{\prime} / u_{b}}$ for comparable Re. " $\bigcirc ” \beta=3.3$, “×” $\beta=6.7$, “ $\square$ ” $\beta=10$. 


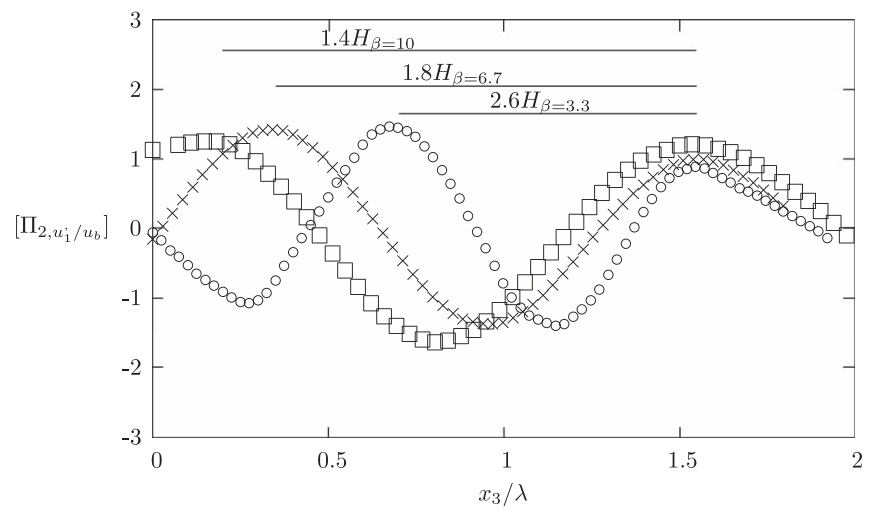

FIG. 9. Second eigenfunctions of $u_{1}^{\prime} / u_{b}, \Pi_{2, u_{1}^{\prime} / u_{b}}$ for comparable $R e$. “ $\bigcirc$ ” $\beta=3.3$, “ $\times$ ” $\beta=6.7$, “ $\square$ ” $\beta=10$.

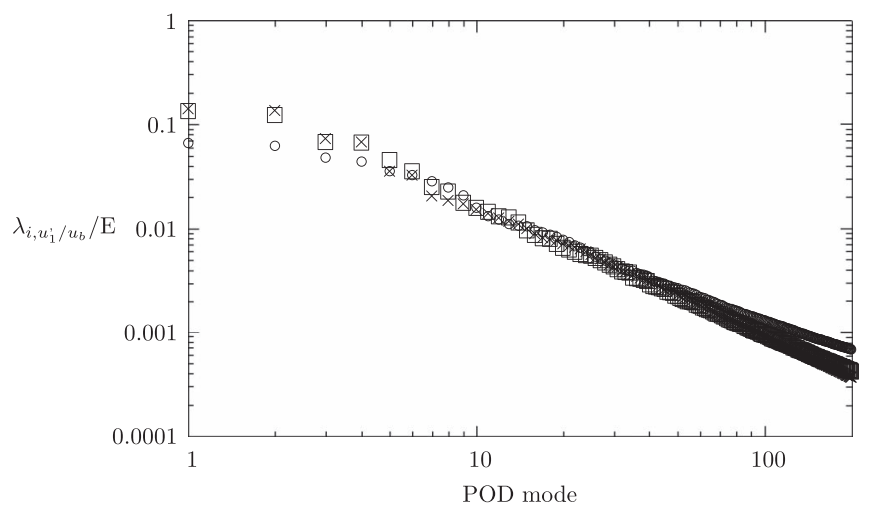

FIG. 10. Eigenvalues of $u_{1}^{\prime} / u_{b}, \lambda_{i, u_{1}^{\prime} / u_{b}}$ for comparable Re. " $\bigcirc ” \beta=3.3$, “×” $\beta=6.7$, “ $\square$ " $\beta=10$.

\section{Characteristic zones}

Sections IV A and IV B highlighted that large-scale structures in flows over wavy walls in narrow channels are influenced by the channel height. In addition, reducing the distance between the flat top and the wavy bottom wall will also influence the characteristic zones of flows over wavy walls. The separation zone (region I) is addressed by comparing mean streamwise velocity profiles at different blockage factors $\beta$ and comparable $R e$ in the vicinity of the wavy bottom along the wave profile (Fig. 11). For this comparison the mean streamwise velocities $\overline{u_{1}}$ are normalized with the friction velocity $u_{\tau}$, a quantity also used by Hudson $e$ e $a l .{ }^{26}$ and Kruse $e t a l .{ }^{17}$ to normalize turbulent quantities in wavy wall flows (for the determination of $u_{\tau}$ for the different cases we refer to the Appendix).

It is observed that the profiles of $\overline{u_{1}} / u_{\tau}$ for $\beta=6.7$ and 10 are nearly identical. Both show reversed flow at $x_{1} / \lambda=0.25$, which clearly indicates the presence of the separation zone. However, at $\beta=3.3$ no separation zone is observed. Compared to $\beta=6.7$ and 10 , the inflection points of the velocity profiles for $\beta=3.3$ at $x_{1} / \lambda=0.25$ and 0.5 are shifted towards the wavy wall. According to Hudson et al.,${ }^{26}$ the region surrounding the inflection points corresponds to the shear layer (regions II and III). The shift of the shear layer towards the wavy bottom wall for $\beta=3.3$ will directly influence the generation and transport of turbulence. Furthermore, $u_{\tau}$ is only confirmed as proper scaling parameter for $\beta=6.7$ and $\beta=10$, since for these two cases the normalized profiles coincide, which is not the case for $\beta=3.3$.

We address the shear layer developing after the wave crest by investigating the distribution of the Reynolds stresses for the different channel heights. Contour plots of the Reynolds stress $\overline{u_{1}^{\prime} u_{2}^{\prime}}$ normalized by $u_{\tau}^{2}$ are presented in Figs. $12(\mathrm{a})-12(\mathrm{c})$. It is observed that with increasing $\beta$ the horizontal extent of the shear layer decreases. For $\beta=3.3$ the shear layer begins to develop at 

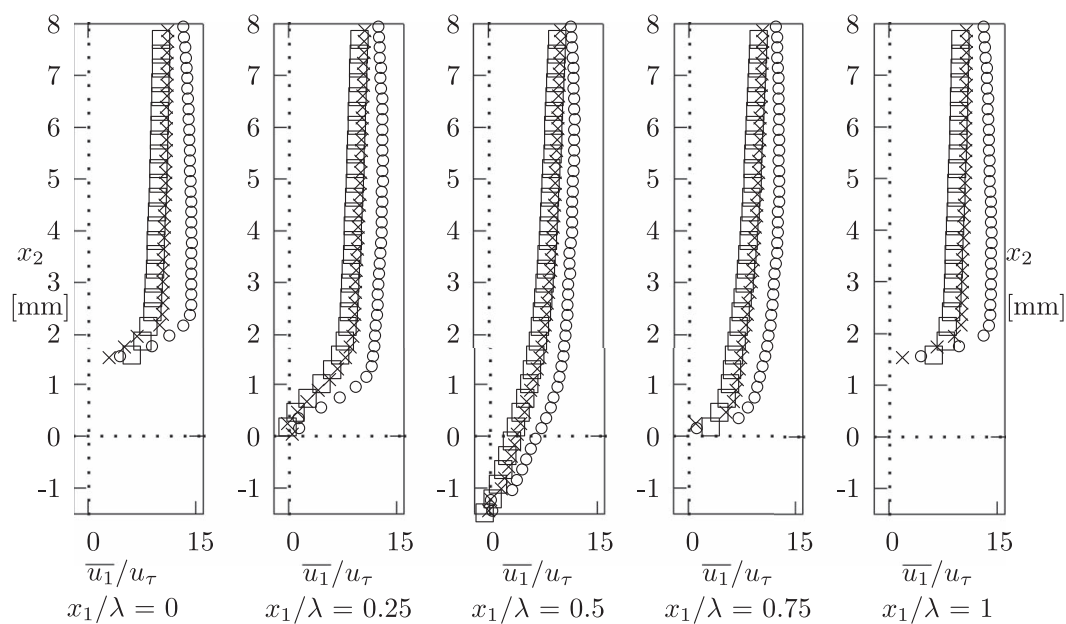

FIG. 11. Normalized mean streamwise velocities $\overline{u_{1}} / u_{\tau}$ at $R e$ around 10000 at three different channel heights. $x_{2}$ is the distance from the wall. " $\bigcirc$ " $\beta=3.3$, “×” $\beta=6.7$, “ $\square$ " $\beta=10$.

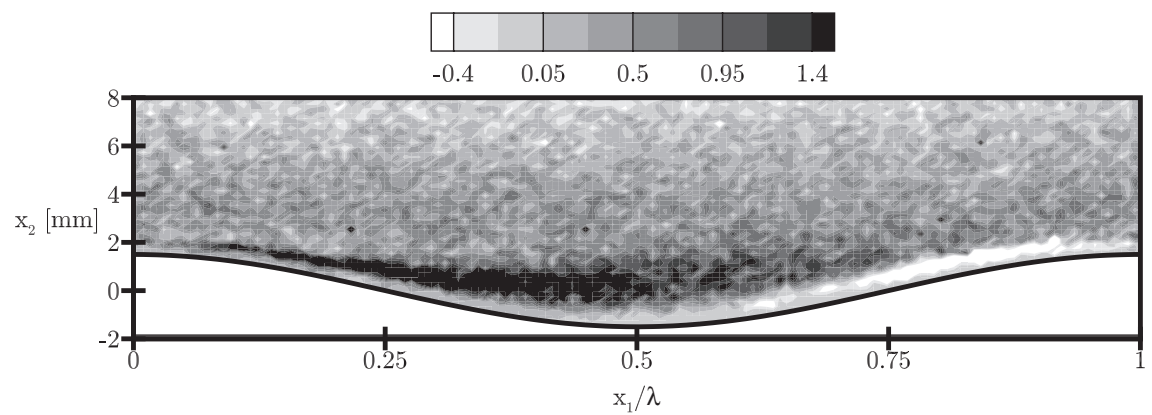

(a)

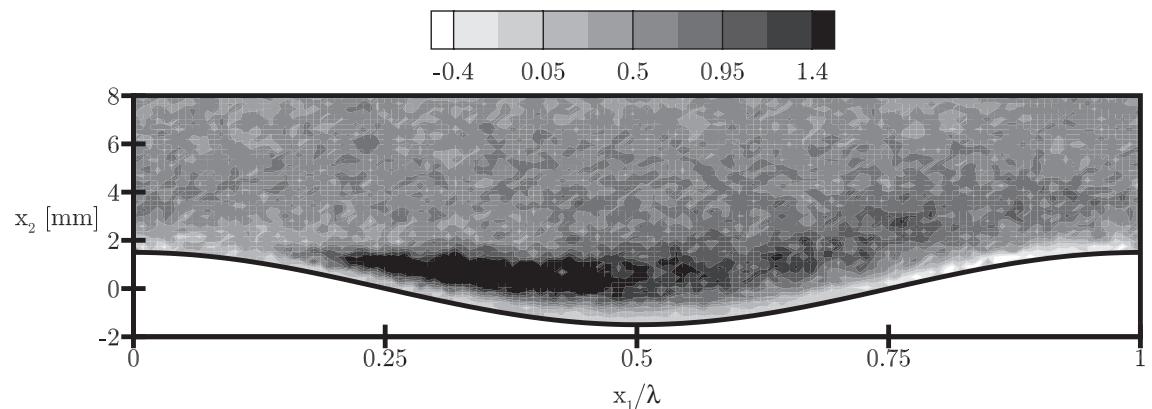

(b)

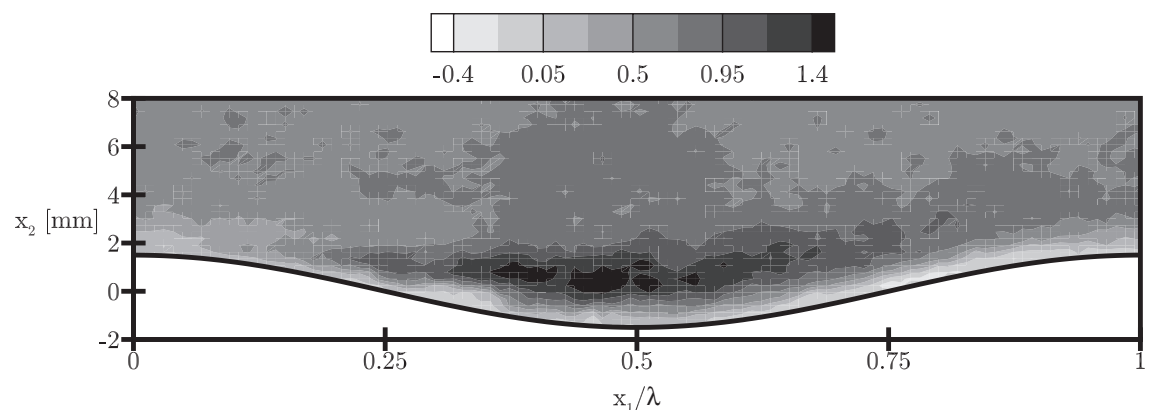

(c)

FIG. 12. (a) $\overline{u_{1}^{\prime} u_{2}^{\prime}} / u_{\tau}^{2}$ at $R e=8600$ at $\beta=3.3$. (b) $\overline{u_{1}^{\prime} u_{2}^{\prime}} / u_{\tau}^{2}$ at $R e=9200$ at $\beta=6.7$. (c) $\overline{u_{1}^{\prime} u_{2}^{\prime}} / u_{\tau}^{2}$ at $R e=10900$ at $\beta=10$. $x_{2}$ is the distance from the wall. 
$x_{1} / \lambda=0.1$, whereas at $\beta=10$ the development starts at $x_{1} / \lambda=0.25$. Furthermore, the smallest vertical extent of the shear layer is found for the case $\beta=3.3$. As already indicated by the inflection points of the mean streamwise velocity profiles $\overline{u_{1}} / u_{\tau}$, the shift of the shear layer towards the wavy bottom wall is also confirmed by the Reynolds stress data.

\section{CONCLUSIONS}

Flows over wavy walls in narrow channels are influenced by the channel height. PODs were performed to characterize the influence of the channel height on the most dominant flow structures. Narrower channels show flow structures with smaller spatial extensions. A common length scale for all three cases was not found. At $\beta=6.7$ and $\beta=10$, the spanwise length scales of the most dominant eigenmodes are $\approx 1.5 H$, whereas for $\beta=3.3$ these spanwise scales increase to $\approx 3 H$. Also the position of the most dominant eigenmode is shifted towards the wavy wall for narrower channels. Here, for $\beta=6.7$ and $\beta=10$ the maximum position of the wavy wall is at $\approx 0.26 H$ from the wavy wall. In the most narrow channel, the maximum is at $0.1 H$ in the wave trough. The eigenvalues of the different cases have shown that the energy is similarly distributed among the flow structures for $\beta=6.7$ and $\beta=10$. In contrast, at $\beta=3.3$ increasingly broader energy spectra are found. The different scaling of the eigenfunctions and the different energy spectra at comparable Reynolds numbers indicate that the structural similarity of large-scale structures does not hold for the smallest blockage ratio. To the authors knowledge such a deviation has never been reported before.

In addition to this blockage effect on the large-scale structures, we also observed an influence on the characteristic zones. For the case $\beta=3.3$ the flow is not separating after the wave crest and compared to $\beta=6.7$ and $\beta=10$ the location of the shear layer is shifted towards the wavy wall.

These results show that the channel height plays an important role for flows over wavy walls. Nakagawa et al. ${ }^{9}$ investigated wavy wall flows in a channel with $\beta=100$ and found that the outer flow can be compared to the outer flow over a flat wall. The investigated system is classified as flows over a rough surface and universality of the outer flow is commonly believed for such kind of flows. Measurements of Kruse et al. ${ }^{17}$ in narrower channels ( $\beta=5$ to 10) showed a universal behavior of the outer flow independent of different wavy wall geometries. This is rather surprising, since the observed flows are considered as flows over obstacles, which are supposed to be dependent on the roughness geometry. This study reveals that the outer flow of an even narrower channel is different compared to wavy channels investigated in the past. The energy spectra of the flow structures get broader and the characteristic length scale of the most dominant flow structures have increased relative to the channel height. Structural similarity at large scales of the outer flow is not anymore valid in smaller dimensions. This finding is not in contradiction to the observed structural similarity of hairpin-like structures and hairpin vortex packets observed by Natrajan et al..$^{23}$ in turbulent capillary and pipe flows. Hairpin vortices are, due to their small length scale, no large-scale structures in our sense.

\section{ACKNOWLEDGMENTS} (SNF).

We gratefully acknowledge the financial support from the Swiss National Science Foundation

\section{APPENDIX: FRICTION VELOCITY}

Hudson et al. ${ }^{26}$ and Kruse et al. ${ }^{17}$ used the friction velocity $u_{\tau}$ to normalize turbulent quantities in wavy wall flows. The good agreement of their measurements of turbulent quantities justifies $u_{\tau}$ as proper velocity scale. The friction velocity is defined as

$$
u_{\tau}=\sqrt{\frac{\tau_{w}}{\rho}}
$$




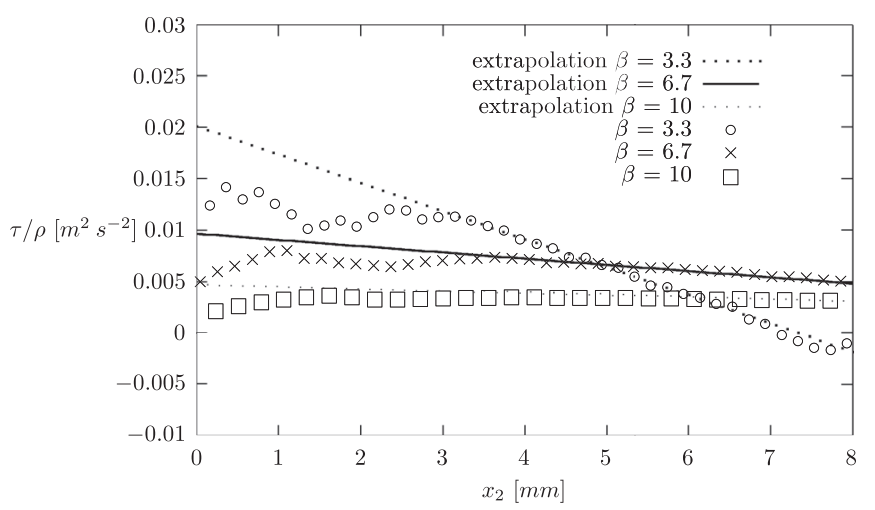

FIG. 13. Streamwise-averaged $\tau / \rho$ at $R e$ around 10000 at three different channel heights. $x_{2}$ is the distance from the wall.

where $\tau_{w}$ is the shear stress at the wall and $\rho$ the density. The shear stress in a turbulent flow over a wavy wall consists of the viscous and the Reynolds shear stress and is given by

$$
\tau=\mu \frac{\partial \overline{u_{1}}}{\partial x_{2}}-\rho \overline{u_{1}^{\prime} u_{2}^{\prime}} .
$$

In the near wall region the viscous stress dominates the flow due to the large velocity gradient and the effect of the no-slip boundary condition on $u_{1}$ and the blockage effects on $u_{2}$. In Fig. 13, friction velocities at $\beta=3.3,6.7$, and 10 and at $R e$ around 10000 are determined from linear extrapolations of streamwise-averaged shear stresses from the outer flow towards the wall. Hudson et al. ${ }^{26}$ proposed this methodology to determine the friction velocity for a flow over a wavy wall, due to the linear variation of the outer flow's shear stresses, which is also present for our cases.

We obtain the following friction velocities: $u_{\tau}=0.14 \mathrm{~m} / \mathrm{s}(\beta=3.3), u_{\tau}=0.098 \mathrm{~m} / \mathrm{s}(\beta=6.7)$, and $u_{\tau}=0.069 \mathrm{~m} / \mathrm{s}(\beta=10)$.

We also applied the bootstrap analysis to the friction velocity by taking into account the normalized shear stresses $\tau / \rho$ in the outer region. The following sampling errors for the friction velocities are obtained: $4.86 \%$ ( $\beta=3.3), 4.29 \%(\beta=6.7)$, and $4.29 \%(\beta=10)$.

${ }^{1}$ T. Stanton, D. Marshall, and R. Houghton, "The growth of waves on water due to the action of wind," Proc. R. Soc. London 137, 283-293 (1932).

${ }^{2}$ H. Motzfeld, "Die turbulente Strömung an welligen Wänden,” Z. Angew. Math. Mech. 47, 193-212 (1937).

${ }^{3}$ D. P. Zilker, G. W. Cook, and T. J. Hanratty, "Influence of the amplitude of a solid wavy wall on a turbulent flow. Part 1. Non-separated flows," J. Fluid Mech. 82, 29-51 (1977).

${ }^{4}$ C. B. Thorsness, P. E. Morrisroe, and T. Hanratty, "A comparison of linear theory with measurements of the variation of shear stress along a solid wave," Chem. Eng. Sci. 33, 579-592 (1978).

${ }^{5}$ P. Cherukat, Y. Na, T. Hanratty, and J. B. McLaughlin, "Direct numerical simulation of a fully developed turbulent flow over a wavy wall," Theor. Comput. Fluid Dyn. 11, 109-134 (1998).

${ }^{6}$ D. S. Henn and R. I. Sykes, "Large-eddy simulation of flow over wavy surfaces," J. Fluid Mech. 383, 75-112 (1999).

${ }^{7}$ D. P. Zilker and T. J. Hanratty, "Influence of the amplitude of a solid wavy wall on a turbulent flow. Part 2. Separated flows," J. Fluid Mech. 90, 257-271 (1979).

${ }^{8}$ M. R. Raupach, R. A. Antonia, and S. Rajagopalan, "Rough-wall turbulent boundary layers," Appl. Mech. Rev. 44, 1-25 (1991).

${ }^{9}$ S. Nakagawa, Y. Na, and T. Hanratty, "Influence of a wavy boundary on turbulence. I. Highly rough surface," Exp. Fluids 35, 422-436 (2003).

${ }^{10} \mathrm{~S}$. Nakagawa and T. Hanratty, "Influence of a wavy boundary on turbulence. II. Intermediate roughened and hydraulically smooth surfaces," Exp. Fluids 35, 437-447 (2003).

${ }^{11}$ C. A. Miller, "Turbulent boundary layer above complex terrain," Ph.D. dissertation (University of Western Ontario, 1995).

${ }^{12}$ W. Gong, P. A. Taylor, and A. Dörnbrack, "Turbulent boundary-layer flow over fixed aerodynamically rough twodimensional sinusoidal waves," J. Fluid Mech. 312, 1-37 (1996).

${ }^{13}$ A. Günther and P. Rudolf von Rohr, "Large-scale structures in a developed flow over a wavy wall," J. Fluid Mech. 478, 257-285 (2003).

${ }^{14}$ N. Kruse, A. Günther, and P. Rudolf von Rohr, "Dynamics of large-scale structures in turbulent flow over a wavy wall," J. Fluid Mech. 485, 87-96 (2003).

${ }^{15}$ A. Günther and P. Rudolf von Rohr, "Structure of the temperature field in a flow over heated waves," Exp. Fluids 33, 920-930 (2002). 
${ }^{16}$ N. Kruse and P. Rudolf von Rohr, "Structure of turbulent heat flux in a flow over a heated wavy wall," Int. J. Heat Mass Transfer 49(19-20), 3514-3529 (2006).

${ }^{17}$ N. Kruse, S. Kuhn, and P. Rudolf von Rohr, "Wavy wall effects on turbulence production and large-scale modes," J. Turbul. 7, 1-24 (2006).

${ }^{18}$ S. Kuhn, C. Wagner, and P. Rudolf von Rohr, "The influence of wavy walls on the transport of a passive scalar in turbulent flows," J. Turbul. 9, 1-17 (2008).

${ }^{19}$ C. Wagner, S. Kuhn, and P. Rudolf von Rohr, "Scalar transport from a point source in flows over wavy walls," Exp. Fluids 43, 261-271 (2007).

${ }^{20}$ S. Kuhn and P. Rudolf von Rohr, "Experimental study of heat flux in mixed convective flow over solid waves," Exp. Fluids 44, 973-984 (2008).

${ }^{21}$ S. Kuhn, S. Kenjereš, and P. Rudolf von Rohr, "Large eddy simulations of wall heat transfer and coherent structures in mixed convection over a wavy wall," Int. J. Therm. Sci. 49, 1209-1226 (2010).

${ }^{22} \mathrm{C}$. Wagner, S. Kenjereš, and P. Rudolf von Rohr, "Dynamic large eddy simulations of momentum and wall heat transfer in forced convection over wavy surfaces," J. Turbul. 12, 1-27 (2011).

${ }^{23}$ V. Natrajan, E. Yamaguchi, and K. Christensen, "Statistical and structural similarities between micro- and macroscale wall turbulence," Microfluid. Nanofluid. 3, 89-100 (2007).

${ }^{24}$ S. Kuhn, C. Wagner, and P. Rudolf von Rohr, "Influence of wavy surfaces on coherent structures in a turbulent flow," Exp. Fluids 43, 251-259 (2007).

${ }^{25}$ L. Sirovich, "Turbulence and the dynamics of coherent structures. Part 1. Coherent structures," Q. Appl. Math. XLV, 561-571 (1987).

${ }^{26}$ J. D. Hudson, L. Dykhno, and T. Hanratty, "Turbulence production in flow over a wavy wall," Exp. Fluids 20, 257-265 (1996). 